\title{
La portée de la comparaison, de la métaphore et de la métonymie sur la continuité référentielle en moyen français
}

\author{
Estèle Dupuy-Parant \\ Centre d'études supérieures de civilisation médiévale (CESCM) \\ parantdupuy@orange.fr
}

\section{Introduction}

Cette étude textuelle diachronique s'inscrit dans le cadre d'une étude plus large (Dupuy-Parant : 2006) sur la continuité référentielle dans la prose française $d u X^{\text {ème }}$ à la fin du XV ème siècle dont l'hypothèse première est que le choix des expressions anaphoriques au sein de la chaîne anaphorique ne relève pas de la subjectivité au sens où ce n'est pas celui qui écrit qui, dans la majorité des cas ${ }^{1}$, décide « consciemment » d'utiliser telle ou telle forme anaphorique (Cornish, 2000). Les notions d'anaphore et de chaîne anaphorique sont au cœur de ce travail ${ }^{2}$ centré sur la personne 3 et 6 en récit. Notre corpus est composé de trois textes $^{3}$ : les Quinze Joyes de Mariage ${ }^{4}$, les Chroniques de Froissart ${ }^{5}$ et les Mémoires de Commynes $^{6}$. Il permet une comparaison triangulaire : diachronique, de genre, voire dialectale ${ }^{7}$. En outre, ces textes présentent trois catégories d'expressions référentielles valables quel que soit le milieu discursif $^{8}$ :

- les anaphores nominales ${ }^{9}$ répondant à deux catégories référentielles différentes - les redéfinitions (anaphore lexicale infidèle, pronom indéfini, déterminant démonstratif suivi d'un nom renommant, anaphore avec déterminant ledict) ${ }^{10}$ et les réinitialisations (anaphore lexicale fidèle propre ou impropre sans ledict $)^{11}$. Les réinitialisations reprennent le même référent en utilisant (exception faite du déterminant ${ }^{12}$ ) le même SN que le précédent. C. Schnedecker présente les réinitialisations comme frontière des énoncés :

\begin{abstract}
« Leurs frontières - et partant leur extension - sont suffisamment nettes et étanches pour empêcher qu'il n'y ait entre eux [= les énoncés] la moindre inter-connexion. En sorte que, la co-référence n'échappant pas à cette protection territoriale, la réinstanciation du référent dans un domaine autre que celui de sa mention originelle impliquerait tout naturellement la redénomination puisqu'elle-même contribue, à un autre plan de l'organisation textuelle, à renforcer cette rupture. [...] entre deux intervalles, il $[=$ le référent $]$ a pu avoir le temps d'évoluer et de changer $[\ldots]$ la redénomination est un moyen commode de suggérer, au plan ontologique, ces ruptures (ou assimilées dans le cas des fictions) » (Schnedecker, 1997 : 132-134)
\end{abstract}

mais ce n'est pas la seule cause de réinitialisation. La réinitialisation est due en majeure partie à l'application des règles syntactico-sémantiques que nous présenterons ultérieurement.

Les redéfinitions mentionnent le référent sous une forme nominale qui n'utilise pas les mêmes lexèmes que la forme nominale précédemment utilisée pour ce référent. Le choix d'une autre forme lexicale permet de présenter le référent selon un axe d'approche différent du précédent. Certains linguistes les appèlent redénominations. Mentionner ce phénomène anaphorique redénomination souligne le changement de forme lexicale. Utiliser le terme redéfinition permet, selon nous, de signaler la conséquence de cette redénomination, à savoir que, sans pour autant qu'il y ait d'évolution du référent même si cela est parfois le cas (une seule occurrence dans le corpus $^{13}$ ), ce référent va être perçu selon un axe référentiel différent de celui que livrait la mention lexicale antérieure.

- les anaphores pronominales réalisées par le pronom personnel, le pronom relatif et le pronom démonstratif seul 
- les anaphores zéro avec l'appui de la désinence verbale qui n'est pas ambiguë en MF.

Vérifiée, cette hypothèse a permis de mettre au jour une combinatoire de cinq règles : l'une dite de concurrence référentielle (désormais C.Rf.) concernée par cette étude; trois dites valentielloréférentielles $^{14}$ qui montrent l'importance de la sémantique verbale première (Blanche-Benveniste: 1984 ) et du chaînage valentiel dans la continuité référentielle et une dernière dite syntactico-valentielle qui conditionne les règles valentiello-référentielles à la nature syntaxique de la proposition dans laquelle entre le verbe dont le référent est argument (Dupuy-Parant : 2006 et 2007).

Pour la continuité référentielle et la règle de C.Rf. en particulier, nous avons observé les retentissements de constructions inter-référentielles - constructions référentielles faisant entrer en relation deux référents - telles que les comparaisons, les métaphores et les métonymies dans le choix des expressions anaphoriques afférentes aux référents concernés. Après une brève présentation de cette règle de C.Rf. et du rôle qu'y joue la sémantique primitive verbale, les implications syntactico-sémantiques et référentielles de chacune de ces trois constructions seront observées.

\section{La règle de concurrence référentielle et la sémantique première du verbe}

En cas de co-présence de référents, l'ambiguïté référentielle naît-elle de l'utilisation de l'anaphore pronominale ou zéro ? L'anaphore nominale qui véhicule des informations sémantiques et référentielles discriminantes, apparaît-elle toujours dans des contextes où l'anaphore pronominale ou zéro auraient pu engendrer une ambiguïté référentielle ? Le fait que nous soyons amenée à formuler ces questions suggère qu'il est légitime de supposer que le choix de l'expression anaphorique peut dépendre de l'existence et du degré de C.Rf. qui existe entre référents co-présents. Nous avons étudié les situations de co-présence de référents, divergeant par une seule de leur caractéristiques référentielles (genre, nombre ou nature sémantique) ou présentant des caractéristiques référentielles identiques ${ }^{15}$. Ces observations nous ont conduite à la règle de C.Rf.(cf. Dupuy-Parant, 2007) :

Lorsque les référents co-présents divergent par une seule de leur caractéristiques référentielles - leur genre ou nombre comme dans (1) ou leur nature sémantique, comme dans (2) entre "bon homme" et "'brouet" - l'anaphore pronominale et/ou zéro sont utilisées sans ambiguité référentielle. Il n'y a donc pas de C.Rf. entre référents de caractéristiques référentielles différentes. La sémantique verbale primitive, seconde, voire ternaire ${ }^{16}$ permettent d'établir une situation de co-présence non ambiguë.

(1) La premiere joye de mariage si est quant le jeune homme est en sa belle jeunesse, qu'il est frois, net et plaisant et ne s'esmoye fors de tirer esgulletes, faire ballades et icelles chanter, regarder les plus belles, et aviser ou il pourra trouver maniere d'avoir ses plaisirs et trouver ses jolivetez selon l'estat dont $\underline{\underline{i}}$ est, et ne s'esmoye point dont $\underline{\mathbf{i}}$ vient, pour ce que a l'aventure il a encore pere et mere ou aultres parens qui lui baillent ce qu'il luy fault. Et combien qu'il a aises et plaisances largement, $\underline{\text { il ne }}$ nes peut endurer, mais regarde les autres mariés qui sont en la nasse bien embarrez, qui s'esbanoient, ce lui semble, pour ce qu'ilz ont l'apast emprés eux dedens la nasse, c'est assavoir la femme, qui est belle, bien paree et bien abille de tieulx abillemens que a l'aventure son mari n' n'a pas paiez, car l'en lui fait acroire que son_pere o ou $\underline{\text { sa }}$ menere les li ont donnez de leur livree. (QJM, Premiere joye, p. 6, 1.1 à 18)

(2) Lors se met le bon homme a la voie, et est cuisiner et s'art a faire le brouet ou se eschaude pour le garder de fumer, et tence sess.gens et dit qu'ilz.ne sont que bestes et qu'ilz ne scevent rien faire. "Vroiement, [...] oncques. » Lors le bon homme s'en va et porte son brouet a la dame et la efforsse et prie tant que elle en prent une partie pour l'amour de $\underline{\text { lui}}$, ce dit elle, en disant qu'il est tres bon et $\underline{\underline{\text { ce }}}$ que les aultres lui

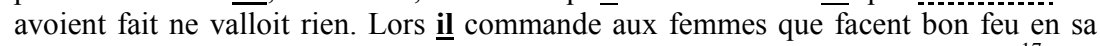
chambre et que elles se tiennent pres elle. (QJM, Tierce joye, p. 22, 1. 136 à 154) ${ }^{17}$

En revanche, la C.Rf. existe entre référents co-présents de caractéristiques référentielles identiques, comme dans (3). Dans ce cas, l'anaphore nominale, plus complète sémantiquement et plus discriminante 
que l'anaphore pronominale ou zéro, est utilisée à la reprise de chaque chaîne anaphorique après interposition du référent d'une autre chaîne anaphorique.

(3) Et offroit ledict connestable prendre Sainct Quentin tous les jours qu'on vouldroit, car ses terres estoient a l'environ; et disoit encores avoir tres grand intelligence en Flandres et en Breban, et qu'il feroit rebeller plusieurs villes contre ledict due [= "duc de Bourgogne'"]. Le duc de Guyenne, qui estoit sur le lieu, et tous ses principaulx gouverneurs offroient fort servir le Roy en ceste querelle et d'amener quatre ou cinq cens hommes d'armes que ledict duc de Guyenne tenoit d'ordonnance. Mais leurs fins n'estoient pas telles que le Roy entendoit, mais toutes a l'opposite, comme vous verréz. (Mém., livre III, chap. I, p. 219, 1. 6-16)

Cette règle de C.Rf. offre peu d'exceptions (tableau 1) qui trouvent par ailleurs une explication à travers les autres règles de la combinatoire ( $c f$. Dupuy-Parant, 2006) :

\begin{tabular}{|c|c|c|c|}
\cline { 2 - 4 } \multicolumn{1}{c|}{} & QJM & Chroniques & Mémoires \\
\hline Anaphores nominales & 11 & 19 & 1 \\
\hline Anaphores pronominales & 5 & 8 & 34 \\
\hline
\end{tabular}

Tableau 1 : Fonctionnements externes à la règle de C.Rf. et impliquant la valence verbale.

Parmi les occurrences étudiées pour la règle de C.Rf., certaines réalisations inter-référentielles particulières dont la comparaison, la métaphore et la métonymie ont retenu notre attention. Ces constructions impliquent la mise en commun d'une ou plusieurs « déterminations » constituant l'horizon de chacun des référents mis en relation (De Mulder, 2001: 236 et Kleiber, 2001:91 à 95). Ainsi, l'horizon d'un référent est constitué de toutes les représentations stéréotypiques (Kleiber, 2001) que peut avoir ce référent c'est-à-dire de toutes ses qualités référentielles et définitionnelles. Lorsque deux référents entrent en relation inter-référentielle sur le mode de la comparaison, de la métaphore ou de la métonymie, une ou plusieurs de leurs qualités/déterminations sont mises en commun (pour la métaphore et la métonymie) ou rapprochées (pour la comparaison). Il est donc légitime de se demander comment ces relations inter-référentielles agissent sur le choix de l'expression anaphorique et si elles ont un impact sur l'application de la règle de C.Rf. ou l'existence même d'une C.Rf..

Le tableau 2 présente leurs occurrences dans notre corpus :

\begin{tabular}{|c|c|c|c|c|}
\cline { 2 - 5 } \multicolumn{1}{c|}{} & QJM $^{18}$ & Chroniques & Mémoires & Total \\
\hline Comparaison & 6 & 3 & 7 & 16 \\
\hline Métaphore & 5 & 1 & 3 & 9 \\
\hline entre comparaison et métaphore & 1 & 0 & 0 & 1 \\
\hline Métonymie & 0 & 1 & 17 & 18 \\
\hline
\end{tabular}

Tableau 2: Occurrences pour lesquelles au moins l'un des référents (comparé ou comparant) est masculin.

\section{La comparaison}

Si pour quatre ${ }^{19}$ de nos occurrences, les référents, comparé et comparant, ne poursuivent pas leur chaîne anaphorique - ce qui ne nous permet pas de tester la règle de C.Rf. -, les 12 autres occurrences apportent de précieuses informations. Deux cas de figure existent: les comparaisons mettant en relation deux référents de caractéristiques référentielles différentes $(6$ occ.) et celles mettant en relation deux référents de caractéristiques référentielles identiques ( 6 occ.). 


\subsection{Référents, comparé et comparant, de caractéristiques référentielles différentes}

Lorsqu'une comparaison se met en place entre deux référents de caractéristiques référentielles différentes, la mise en commun de l'une des informations « stéréotypiques » (Kleiber 2001 : 91 à 95) composant leur horizon permet de les identifier l'un à l'autre. D'après la règle de C.Rf., la divergence de caractéristiques référentielles entre eux élimine la C.Rf. d'où l'utilisation de l'anaphore pronominale ou zéro à la reprise de leur chaîne anaphorique respective. La relation de comparaison ne modifie pas cette constante. Toutes les occurrences relevées voient leur comparant et/ou leur comparé poursuivre leur chaîne anaphorique par anaphore pronominale ou zéro sans ambiguité référentielle, comme dans (4) et (5) où la chaîne anaphorique du référent comparé se poursuit par anaphore pronominale :

(4) Et lors pense le pouvre homme nouvel mesnagier, qui a a faire moult de chouses, qui a l'aventure la roube coustera .L. ou .LX. escuz d'or. Et en pensant il ne trouve pas bien maniere d'avoir chevanche et toutevoies il la lui fault avoir, car $\underline{\mathbf{l}}$ voit $\underline{\underline{\mathbf{s a}}}$ $\underline{\underline{\text { femme }}}$ que est telle a son avis qu'il loue $\underline{\text { Dieu }}$ en son courage dont $\underline{\underline{i}}$ lui donna ung si riche joyau come el est. (QJM, Premiere joye, p. 11, 1. 181 à 189)

Idem pour: QJM, Quinte joye, p. 36, 1. 101 à 110 : "la dame" comparée à "un tasteur de vins" puis reprise par anaphore pronominale, elle; QJM, Quinte joye, p. 44, 1. 400-403: "la dame" comparée à "ung ymage" (divergence de nature sémantique) puis reprise par anaphore pronominale, elle.

(5) Et avint que [...], li rois de France [...] dist que il ne voloit fors guerriier des gentils honmes dou roiaume de France, et que des conmunautés amener en bataille,

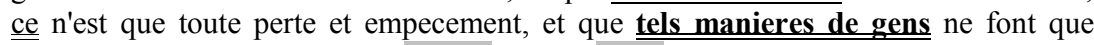
fondre en bataille ensi conme la nive font au solel; et bien avoit aparu a la bataille de Crechi, a la Blanqe Taqe, a Kem en Normendie et en tous les lieus ou on les avoit menés, et que plus il n'en voloit nuls avoir, [...] villes. (Chr., livre I, chap. CCXLVIII, p. 820-821, 1. 24-35)

Idem pour: QJM, Quarte joye, p. 28, 1.32-41.

L'extrait (5) allie métaphore et comparaison. Par métaphore "tels manieres de gens" est mis en relation avec "quelque chose de sensible à la chaleur et qui sous son effet peut devenir liquide" (comme la "neige") à travers le sémantisme du verbe fondre. Cette métaphore est renforcée par comparaison entre les référents comparés tels manieres de gens et bataille et leur comparant respectif la nive et solel (le verbe fondre étant de surcroît repris dans la proposition comparative, font). L'absence de C.Rf. est confirmée suite à la métaphore et la comparaison par l'anaphore pronominale les régime du verbe avoit menés - reprenant le comparé tels manieres de gens.

Dans (6), ce sont les chaînes anaphoriques du comparé "le bon homme" et du comparant "ung cheval recreu" qui se poursuivent par anaphore pronominale avec il. Ce pronom est astucieusement mis en facteur commun des verbes et périphrase verbale coordonnés trote et aille pour gouverner pour référer à leur agent sémantique. Or, le verbe trote (1. 46) appartient au champ lexical de l'équitation et suppose, de par sa sémantique première, un agent sémantique teinté du trait «- personnel» et de par sa sémantique seconde, un agent sémantique teinté du trait «+ animal». Le syntagme verbal aille pour gouverner, à l'inverse, a nécessairement un agent sémantique teinté du trait "+ personnel" en sémantique première et “+ humain" en sémantique seconde - l'homme étant seul apte à "s'occuper de quelque chose". Le pronom personnel il (1.46) renvoie donc à deux référents distincts ce qui permet notamment d'assurer la continuité référentielle de la chaîne anaphorique principale, réalisant ainsi une métaphore "filée".

(6) Et pour ce, lui, voiant les chargez dessus dites et ce qu'il a a faire, come j'ay dit, il ne lui chault mes qu'il vive, et est tout en non chaloir, comme ung cheval recreu qui ne fait compte de l'esperon ne de chouse que l'en lui face. Ce non obstant, il faut qu'il trote et aille par païs pour gouverner sa terre ou pour sa marchandise, selon l'estat dont il est. (QJM, Quarte joye, p. 28, 1. 41 à 48)

Idem pour QJM, Quarte joye, p. 27, 1. 1à 8: L'anaphore nominale le pouvre homme suite à sa comparaison au référent "ung veil asne" ne découle pas de la C.Rf. mais d'une redéfinition liée à l'évolution contextuelle. 
Diachronie, histoire de la langue

DOI $10.1051 / \mathrm{cmlf0} 039$

\subsection{Référents, comparé et comparant, sont de caractéristiques référentielles identiques}

D'après la règle de C.Rf., la co-présence de référents de mêmes caractéristiques référentielles entraîne une C.Rf. qui induit l'utilisation de l'anaphore nominale à la reprise de la chaîne anaphorique de l'un ou l'autre de ces référents. Or, en situation de comparaison, il semble que la C.Rf. s'efface entre les référents comparé et comparant. Ainsi l'anaphore nominale n'est pas nécessaire à la reprise de l'un ou l'autre de ces référents suite à l'établissement de la comparaison - nous verrons également ci-après qu'il faut prendre en compte les constructions syntaxiques qui expriment les comparaisons lorsque celles-ci introduisent la comparaison en proposition subordonnée. Ainsi, le référent comparé peut poursuivre sa chaîne anaphorique par anaphore pronominale ou zéro sans que la mention intermédiaire du référent comparant ne perturbe la continuité référentielle de sa chaîne anaphorique. Il en va ainsi pour (7), (8) et (9) :

(7) Et tantoust viennent les commeres et le proudomme va au davant, qui les festoye et fait bonne chiere, et est sans chapperon par la meson, tant est jolis, et semble ung foul combien qu'il ne l'est pas. (QJM, Tierce joye p. 25, 1. 262 à 266)

(8) Qui eust peu prandre partie des condictions du Roy nostre maistre et partie des siennes [ [ 'duc de bourgogne'], on en eust bien fait ung prince parfaict, car, sans nulle doubte, le Roy en sens le passoit de trop, et la fin l'a monstré par ses oeuvres. (Mém., livre III, chap. III, p. 229, 1. 39 - p. 230, 1. 2)

(9) L'armee dudict duc de Bourgongne estoit plus forte par mer que celle du Roy et dudict conte ensemble, car il avoit prins au port de l'Escluse largement grosses navyres d'Espaigne et de Portingal, deux naves de Gennes et plusieurs hulques d'Alemaigne. Le roy Edouard n'estoit point homme de grand ordre, mais fort beau prince, plus que nul que j'aye veu jamais en ce temps la, et tres vaillant. II ne se soucioyt point tant de la descente dudict conte comme faisoit le duc de Bourgongne, lequel sentoit des mouvemens par Angleterre en faveur dudict conte de Warvic, et en advertissoit souvent ledict roy ; mais $\underline{\underline{\mathbf{i}}}$ n'avoit nulle craincte, (Mém., livre III, chap. V, p. 235, 1. 39 - p. 230,1.2) Extrait regroupant 3 des 6 occ. chez Commynes. Idem pour Mém., livre III, chap. V, p. 240, 1. 11-13.

Dans (7), la C.Rf. n'apparaît pas - reprise du référent "le prudomme" par anaphore pronominale - sans doute parce que la comparaison n'est pas continue mais aussi, parce que le lexème ung foul peut être l'une des «représentations stéréotypiques » du référent "le prudomme" et que le sémantisme du verbe semble induit une idée de compraison mais aussi de démenti.

Pour (8), le référent "le Roy" entre en comparaison avec le référent 'duc de Bourgogne' - repris par le pronom personnel régime direct, $l e-\mathrm{du}$ fait du sens intensif de l'adverbe trop. Après comparaison, la chaîne anaphorique du référent "Roi" se poursuit par anaphore zéro avec la personne 3 du verbe $a$ monstré.

Ces deux exemples soulignent aussi le fait que la comparaison peut naître de phénomènes syntaxiques assez différents mais que, quels qu'ils soient, elle ne semble pas perturber la C.Rf. de la chaîne anaphorique du comparant.

L'extrait (9) permet de faire une remarque importante sur les modalités d'expression de la comparaison qui peut induire ou non une subordination. Dans (9), les comparaisons utilisent les outils comparatifs syntaxiques subordonnants tels que plus/autant/myeulx ... que et comme. Or dans le cas d'une comparaison avec subordination (Melis, 1994), viennent s'ajouter à la règle de C.Rf., les autres règles de la combinatoire à savoir les règles valentiello-référentielles et syntactico-valentielle ( $c f$. Dupuy-Parant, 2007 et 2006 $)^{20}$. La proposition subordonnée comparative fait partie des propositions à frontière de sortie étanche ( $c f$. note 20) c'est-à-dire que la répartition des référents sur les arguments de son verbe n'influe pas sur le choix de l'expression anaphorique du/des référents des arguments du verbe qui suit. Ainsi, pour la comparaison entre le référent "Le roy Edouard" agent sémantique du verbe estoit et le référent comparant indéfini " "nul que j'aille veu jamais en ce temps la", l'anaphore pronominale, $i l$, sujet et agent sémantique de soucioyt montre d'une part la continuité valentiello-référentielle entre propositions non 
régies appuyée par l'anaphore pronominale, et, d'autre part, que la présence intermédiaire d'un référent comparant ne provoque pas de C.Rf. ${ }^{21}$. Ce processus se répète : le référent "roi Edouard", mentionné par, il comme agent sémantique du verbe soucioyt de proposition non régie est repris par anaphore pronominale, il, comme agent sémantique de n'avoit nulle craincte après l'interposition du référent comparant mentionné par l'anaphore nominale le duc de Bourgongne en subordonnée comparative.

Pour conclure, la comparaison entre deux référents n'engendre pas de C.Rf. entre les deux référents comparés même s'ils sont de mêmes caractéristiques référentielles. Les référents comparés poursuivent leur chaîne anaphorique sans ambiguité référentielle par anaphore pronominale ou zéro malgré l'interposition du référent comparant. De plus, lorsque la comparaison se fait en proposition subordonnée comparative, la distribution valentiello-référentielle de cette subordonnée ne peut franchir sa frontière de sortie pour influer sur celle du verbe suivant de sorte que l'interposition du comparant ne peut pas perturber la chaîne anaphorique du comparé.

\section{La métaphore}

La métaphore (Moeschler et Reboul, 1994) procède à la comparaison entre deux référents en ne mentionnant pas forcément le comparant sous forme lexicale ou nominale : elle peut s'appuyer sur des verbes ou d'autres termes qui en général ne sont attribués qu'au comparant, en les appliquant au comparé. De plus, nous noterons que les référents qui entrent en relation métaphorique n'ont pas les mêmes caractéristiques référentielles, ce qui met de côté la possibilité d'une C.Rf. induisant l'anaphore nominale. C'est le cas de toutes les occurrences relevées dans le corpus qui permettent en outre de distinguer deux types de métaphores : celles in praesentia (10) et celles in absentia (11) et (12) et (13).

Dans l'extrait (10), le référent féminin singulier "ceste place" qui réfère au "port de Calais" est comparé par métaphore in praesentia (la relative qui introduit le comparant signalant en outre explicitement la coréférence) aux référents "le plus grand tresor [...] de la crestienté" : cette mise en commun de l'une de leur "détermination stéréotypique » induit la possibilité de parler de l'un pour évoquer l'autre sans C.Rf. et permet la reprise du référent métaphorisé par l'anaphore pronominale $y$ dans la proposition non régie suivante sans ambiguïté.

(10) il est a penser qu'il [= conte de Warvic] pensoit bien [l!'] [= 'seigneur de Vaucloe'] avoir pourveu en ceste place [= Callais], qui est le plus grand tresor d'Angleterre et la plus belle cappitanerie du monde, a mon advis, au moins de la crestienté. Car je y fuz plusieurs foiz durans ces differans (Mém., livre III, chap. IV, p. 233, 1.13-17)

Pour les métaphores in abstentia (11) et (12), l'anaphore contextuelle Ung mal ne ung peril de (11) et le référent in abstentia de la métaphore filée de (12) ne donnent pas suite à une chaîne anaphorique ce qui ne nous permet pas de tester la règle de C.Rf. :

$(11)^{22} \underline{\text { Les Oustrelins }}$ vindrent semblablement encrer assés pres de luy $[=l e$ 'roi Edouard'], en intencion de le joindre a la maree prouchaine. Ung mal ne ung peril ne vient jamais seul. La fortune de ce Roy estoit bien changee et ses pansees. (Mém., livre III, chap. V, p. 240, 1.28-32)

$(12)^{23}$ Or voiéz ung peu comme les affaires ou brouilliz de ce royaulme sont grands (ainsi se peuvent bien appeller par aulcun temps quant il est en discord) et comme $\underline{\text { ilz }}$ soient presentz et mal aiséz a conduyre et loing de fin quant $\underline{\text { ilz }}$ sont commancéz; car encores qu'ilz ne soient au commancement que deux ou trois princes, ou encores moindres personnaiges, avant que ceste feste ait duré deux ans, tous les voisins y sont conviéz. (Mém., livre III, chap. VIII, p. 254, 1. 31-38) 
En revanche, dans (13), les chaînes anaphoriques des référents métaphorisés se poursuivent. Le groupe référentiel d'entités masculines animées humaines, les autres mariés, est comparé métaphoriquement au "poisson" par le biais des termes l'apast et nasse (champ lexical de la pêche) et de la locution verbale sont en la nasse dont le sémantisme premier « - personnel » et second « - humain /+ animal » identifient ce groupe référentiel au banc de poisson.

(13) Et combien qu'il a aises et plaisances largement, il ne les peut endurer, mais regarde les autres mariés qui sont en la nasse bien embarrez, qui s'esbanoient, ce lui semble, pour ce qu'ilz ont l'apast emprés eux dedens la nasse, c'est assavoir la femme, qui est belle, bien paree et bien abille de tieulx abillemens que a l'aventure

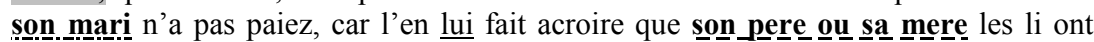
donnez de leur livree. Si tournoye et serche le jeunes homs environ la nasse et fait tant qu'il entre dedens et se marie. Et pour la haste qu'il a de taster du past avient souvent qu'il enquiert petitement des besoingnes et s'i boute tel feur telle vente.

Or est dedens la nasse le pouvre homs qui ne se souloit esmoier fors de chanter, d'echapter esguilletes, bources de saye et autres jolivetés pour donner aux belles. Il se joue et delite ung pou de temps liens [...] (QJM, Première joye, p. 6, 1.9 à 28)

Cette métaphore filée se répercute sur l'animé humain masculin singulier, le jeunes homs, avec le verbe tournoye et le lexème la nasse; "jeunes homs" apparaît également comme agent sémantique de la locution verbale qu'il entre dedens explicitée par la coordination du verbe se marie et la phrase Or est dedens la nasse le pouvre homs. Le référent est donc assimilé à un poisson. Le référent comparant n'est que suggéré par les termes lexicaux utilisés se rapportant à la pêche sans provoquer de C.Rf. : la chaîne anaphorique des référents comparés se poursuit par anaphore pronominale avec la personne 6 , ilz, pour le groupe référentiel, et la personne 3, il, pour le référent le pouvre homs. De plus, la locution, c'est assavoir, explicite la métaphore en stipulant l'identité référentielle de l'apast par l'anaphore nominale, la femme.

Dans les autres extraits ${ }^{24}$, il en va de même: les référents, comparé ou comparant, ne sont pas explicitement mentionnés et de ce fait, la C.Rf. ne semble pas exister puisque la chaîne anaphorique du comparé, si elle se poursuit, peut le faire par anaphore pronominale.

Pour conclure, si la métaphore est in abstentia, le seul référent mentionné sous forme nominale et/ou pronominale est le référent comparé. De fait, la chaîne anaphorique qui en découle réfère au comparé et par métaphore au comparant suggéré à travers leur «détermination stéréotypique » commune. Si la métaphore se fait in praesentia, la « détermination stéréotypique » commune entre comparé et comparant annule la C.Rf. entre eux : ils poursuivent leur chaîne anaphorique par anaphore pronominale ou zéro.

\section{La métonymie (Zribi-Hertz, 1996 et Riegel, 1994)}

Seule (14) relevée chez Froissart présente un référent indéfini coers qui réfère par métonymie synecdoctique in absentia au référent indéfini, "homme". Ce référent indéfini poursuit sa chaîne anaphorique par anaphore pronominale avec les pronoms, personnel, il, et relatif, qui.

(14) Qant $\underline{\underline{i}}$ oirent ce raport, $\underline{\underline{i}}$ conmenchierent tout et toutes a criier et a plorer si tenrement et si amerement que il ne fust si durs coers ou monde, se il les veist et oist $\underline{\underline{\text { euls }}}$ demener, qui n'en euist pité. (Froissart, livre I, chapitre CCLV, p. 842-843, 1. 2532)

Pour les autres occurrences, in praesentia, relevées chez Commynes, deux cas de figure se dessinent : la relation inter-référentielle est synecdoctique ou métonymique.

En relation synecdoctique (6 occ./17), extrait (15), aucun des référents synecdoctiques - la couronne, son esperit, sa personne $e^{25}-$ ne donne suite à une chaîne anaphorique :

(15) Le Roy [...] n'y appella que gens nomméz et qu'il pensoit qui ne contrediroient pas a son vouloir. Et la feit remonstrer plusieurs choses entreprinses que ledict dupc de 
Cette absence de chaîne anaphorique révèle sans doute que le référent synecdoctique - la personne, l'esperit ou la couronne - n'étant que partie d'une entité référentielle animée humaine co-présente ne peut prétendre au même statut référentiel que l'entité animée humaine-source, ni être considérée comme entité à part entière.

En relation métonymique (11 occ./17), le référent métonymique, qui poursuit sa chaîne anaphorique (1 exception $^{26}$ ), entre en relation avec le référent suggéré sans la nécessité d'une anaphore nominale intermédiaire lorsque le référent suggéré refait surface. Ainsi, le référent suggéré peut apparaître directement sous forme pronominale ou zéro sans ambiguité, y compris lorsqu'il y a une différence de genre ou de nombre entre le référent métonymique et le référent suggéré. C'est le cas pour (18) où la relation de métonymie appuyée sur la sémantique primitive et seconde du verbe se tournassent, n'implique pas d'anaphore nominale lors de la mention du référent suggéré "les gens des villes" mentionnés par le pronom personnel ilz:

(18) L'an MCCCCLXX print vouloir au Roy de se revancher du duc de Bourgongne, et luy sembla qu'il en estoit heure ; et secretement traictoit et souffroit tracter que les villes estants sur la riviere de Somme, comme Amyans, Sainct Quentin et Abeville, se tournassent contre le duc et qu'ilz appellassent ses gens d'armes et les missent dedans. (Mém., livre III, chapitre I, p. 217, 1. 13-19)

Idem pour : Mém., livre III, chap. III, p. 226, 1. 24-28; Mém., livre III, chap. I, p. 217, 1. 25-27 (ces villes).

Cependant, l'utilisation de l'anaphore nominale n'est pas exclue au moment du glissement du référent métonymique au référent suggéré et dans le cas de (19), elle permet d'asseoir la relation métonymique par l'intermédiaire de la forme nominale une partie de la ville (sous-groupe référentiel du groupe référentiel ceulx d'Amyens) sémantiquement plus explicite et agent sémantique du verbe tenoit qui admet, en sémantique primitive, un agent sémantique marqué du trait '+ personnel' :

\begin{abstract}
(19) Toutesfoiz, avec ce petit de gens qu'il peult amasser, il [= duc de Bourgogne] tira a Dorlens avecques quatre ou cinq cens chevaulx seullement, en intencion de garder Amyens de tourner ; et la fut cinq ou six jours que ceulx d'Amyens marchandoient, car l'armee du Roy estoit auprés, qui se presenta devant la ville. Et ung coup la refuserent, car une partie de la ville tenoit pour ledict duc, lequel y envoya faire son logis. Et s'il eust eu gens pour y oser entrer en personne, il ne l'eust jamais perdue, mais il n'y ousoit entrer mal acompaigné, combien qu'il en fust requis de plusieurs de la ville. (Mém., livre III, chap. II, p. 222, 1. 2-12)
\end{abstract}

Puis le glissement inverse se fait à nouveau par anaphore nominale de plusieurs de la ville qui suit la mention sous forme pronominale du référent "ville" non métonymisé.

Il en va de même lorsque le référent métonymique apparaît dans le rôle de patient ${ }^{27}$, la sémantique primitive du verbe exerçant également son influence sur ce rôle valentiel.

Ainsi, les relations métonymiques engendrées par la sémantique primitive du verbe colorant le rôle valentiel dans lequel apparaît l'expression anaphorique du référent, induisent la possibilité d'extraire le référent suggéré directement par anaphore pronominale ou zéro sans passer par une étape intermédiaire qui consisterait à présenter ce référent sous une forme nominale plus explicite. La différence de genre ou de nombre entre le référent métonymique et le référent suggéré ne nuit pas à la continuité référentielle qui ne prend appui que sur la relation métonymique existant entre ces deux référents.

\title{
6 Conclusion
}

Cette étude nous aura donc conduite à observer une liberté relative prise par rapport à la règle de C.Rf. lorsque la relation entre référents co-présents est basée sur la comparaison, la métaphore ou la métonymie. Ces trois formes de mise en commun d'une "dimension stéréotypique » conduisent à la 
disparition de la C.Rf., y compris lorsque ces référents co-présents sont de caractéristiques référentielles identiques: l'anaphore pronominale ou zéro peuvent donc poursuivre la chaîne anaphorique du comparant ou du comparé et la continuité référentielle reste intacte ! Cela est rendu possible grâce à la sémantique verbale, au chaînage valentiello-référentiel (doublant le statut thématique) et au fonctionnement syntactico-référentiel qui jouent un rôle décisif en unissant ces référents par l'une de leurs qualités référentielles dans une relation sémantique forte.

\section{Références bibliographiques}

Blanche-Benveniste, Cl., Deulofeu, J., et alii (1984). Pronoms et syntaxe : l'approche pronominale et son application au français. Paris : S.E.L.A.F.

Charolles, M. (1990). L'anaphore associative. Problèmes de délimitation. Verbum, XIII, 3, 119-148.

Cornish, F. (2000). L'accessibilité cognitive des référents, le centrage d'attention et la structuration du discours : une vue d'ensemble. Verbum, XXII, 1, 7-30.

De Mulder, W. (2001). Une approche "Huserlienne" de la continuité référentielle. In: Les référents évolutifs entre linguistique et philosophie: Actes des Journées d'étude des 11 et 12 septembre 1997, De Mulder, W. \& Schnedecker, C. (eds). Recherches linguistiques, 24, 217-241. Paris : Klincksieck.

Dupuy-Parant, E. (à paraître). Le verbe au cœur de la continuité référentielle : Unité structurante et maillage interverbal des systèmes valentico-référentiels. Actes du Colloque international «DIACHRO-3: Evolutions en français », 20, 21 et 22 sept. 2006. Paris : Peter Lang. (coll. « Sciences pour la communication »)

Dupuy-Parant, E. (2007). Les expressions anaphoriques : fréquence et contraintes linguistiques en récit dans les chaînes anaphoriques du XIV ${ }^{\text {ème }}$ au $\mathrm{XV}^{\text {ème }}$ siècle. In: Vanderheyden, A., De Mulder, W., Mortelmans, J. \& Venckeleer, Th. (éds.) (2007). Texte et discours en moyen français, Actes du XI ${ }^{\mathrm{e} m e}$ Colloque international sur le moyen français. Turnhout : Brepols. (coll. « Texte, codex et contexte $3 »), 11-24$.

Dupuy-Parant, E. (2006). La continuité référentielle en moyen français : règles syntactico-sémantiques, thèse de doctorat. Université du Maine, Le Mans.

Givón, T. (1983). Topic Continuity in Discourse : an Introduction. In: Topic Continuity in Discourse. A Quantitative Cross-Language Study, T. Givón (ed.). Amsterdam : J. Benjamins Publishing Company, pp. 1-41.

Grosz, B., Weinstein, S. et Joshi, A. K., (1995). Centering : a framework for modeling the local coherence of discourse. Computational Linguistics, 21, 2, 203-225.

Gundel, J.-K., Hedberg, N. et Zacharski, R. (1993). Cognitive status and the form of referring expressions. Language, 69, 2, 274-307.

Kleiber, G. (2001). L'anaphore associative. Paris : PUF.

Melis, L. (2003). La préposition en français. Paris : Ophrys.

Melis, L. (1998). Les objets : relations grammaticales et rôles sémantiques, D. Willems et L. Melis (éds). Bruxelles : Duculot.

Melis, L. (1994). La typologie des subordonnées circonstancielles et les comparatives. Travaux de linguistique, 27, 97-113.

Moeschler, J. et Reboul, A. (1994). Langage et pertinence: référence temporelle, anaphore, connecteurs et métaphore. Presse Universitaire de Nancy.

Reichler-Beguelin, M.-J. (1988), Anaphore, cataphore et mémoire discursive. Pratiques, 57, p. 15-43.

Riegel, M. (1994). Article défini, anaphore intraphrastique et relations partie-tout. In : Schnedecker, C., Charolles, M., Kleiber G. et David J. (eds), L'anaphore associative, aspects linguistiques, psycholinguistiques et automatiques, 233-250.

Schnedecker, C. (1997). Nom propre et chaînes de référence, Recherches linguistiques, XXI. Paris : Klincksieck. 
Schnedecker, C. et alii (1991). L'anaphore associative : aspects linguistiques psycholinguistiques et automatiques : Synthèse des travaux réalisés dans le cadre du projet PIR-CNRS "Cognisciences" l'anaphore et son traitement. Paris : Klincksieck.

Zribi-Hertz, A. (1996). L'anaphore et les pronoms : une introduction à la syntaxe générative. Villeneuve-d'Ascq (Nord) : Presses universitaires du Septentrion.

\footnotetext{
${ }^{1}$ Excepté peut-être en ce qui concerne les redéfinitions dont nous allons reparler plus loin dans cet article.

${ }^{2}$ La notion de co-référentialité intervient de façon ponctuelle; la continuité référentielle n'est pas remise en cause dans cette étude : les occurrences d'ambiguïté référentielle sont extrêmement rares.

${ }^{3}$ Références du corpus : 5 premières joies des $Q J M$ (Droz, 1967), chap. 248 à 256 inclus du livre I des Chroniques de Froissart (Droz, 1972), et chap. 1 à 8 inclus du livre III des Mémoires de Commynes (Lettres gothiques, 2001).
}

${ }^{4}$ Les Quinze joies de mariage (désormais $Q J M$ ) datent de la fin du XIV ${ }^{\text {ème }}$ siècle.

${ }^{5}$ Les Chroniques de Froissart (désormais Chr.) ont été rédigées vers 1400.

${ }^{6}$ Les Mémoires de Commynes (désormais Mém.) datent de la fin du XV ${ }^{\text {ème }}$ siècle.

${ }^{7}$ Toutefois, nous ne nous y hasarderons pas : dans le cas de Commynes, l'opposition dialectale n'apporte pas de véritables résultats car les textes (mss. de l'extrême fin du XV ${ }^{\text {ème }}$ siècle et du début du XVI ${ }^{\text {ème }}$ siècle et éds. de la première moitié du XVI ${ }^{\text {ème }}$ siècle) ont perdu toute coloration dialectale.

8 « Milieu » est à prendre au sens écologique du terme, par métaphore.

${ }^{9} \mathrm{Y}$ sont incluses les anaphores réalisées par un pronom indéfini. Réalisées par un pronom, elles peuvent néanmoins dans certains cas (fréquemment) apporter une information sémantique que n'apportait pas l'expression nominale coréférente précédente ou avoir une valeur résomptive qui fait écho à sa précédente utilisation co-référentielle. En revanche, quand l'indéfini sert à l'anaphore indirecte ou qu'il est nécessaire pour sa complétude référentielle de référer au contenu sémantique d'un SN co-référent antérieur, il entre dans la catégorie des anaphores pronominales.

${ }^{10}$ Sont incluses les anaphores indirectes ou associatives (Kleiber, 2001, Charolles, 1990 et Schnedecker, 1991), assez rares. Les redéfinitions, dans la majorité des cas, modifient la visée référentielle antérieure. Appliquées au référent en rôle d'agent sémantique ou de patient, elles assurent un apport informationnel nouveau pour le référent, très souvent lié au contexte situationnel.

${ }^{11}$ Les réinitialisations ou « re-nominations » chez certains linguistes évitent l'ambiguïté référentielle.

12 D'où la distinction entre anaphore lexicale propre (lorsqu'il y a identité totale d'une expression anaphorique nominale à l'autre) et anaphore lexicale impropre (lorsque le déterminant diverge d'une expression anaphorique nominale à l'autre).

${ }^{13}$ QJM, Premiere joye: p. 6, 1. 18-27: Si tournoye et serche le jeune homs environ la nasse et fait tant qu'il entre dedens et se marie [...] Or est dedens la nasse le pouvre homs. Sur le référent évolutif : Kleiber, 1994 et 1997, 121122 et Charolles \& Schnedecker, 1993

${ }^{14}$ Ce terme apparaît sous la forme du néologisme valentico-référentiel dans Dupuy-Parant (2006).

${ }^{15}$ Les caractéristiques référentielles inhérentes au référent, déterminées antérieurement, correspondent à un ensemble de traits morphologiques et sémantiques qui caractérisent l'expression référentielle, voire le référent (pour ce qui est des traits sémantiques) : le genre, le nombre en lien avec la personne verbale morphologiquement non-ambiguë en MF et la nature sémantique du référent (animé/inanimé, etc.). Tout référent est définissable d'après ces trois principales caractéristiques référentielles.

${ }^{16}$ La sémantique complexe du verbe, lorsqu'il est actualisé, influe en profondeur sur la référenciation des référents (mentionnés à travers leur expression référentielle) qui viennent prendre appui sur ses rôles valentiels. «Nous entendons par sémantique du verbe :

- la sémantique primitive selon l'approche de Cl. Blanche-Benveniste (1984) au sens où le verbe teinte de traits sémantiques les paradigmes de ces rôles valentiels, le rôle d'agent sémantique (celui sans qui le procès ne peut être réalisé et qui initialise le système de valence du verbe, dit aussi actant 1) et le rôle de patient principal (celui ou la chose pour qui ou pour laquelle le procès est réalisé et qui vient en second dans le système de valence du verbe, dit aussi actant 2). Le système de valence verbale, qui participe de l'actualisation du procès, sert ensuite de support aux 
expressions anaphoriques (nominale, pronominale ou zéro) qui ancrent le procès dans une réalité. Ces deux rôles valentiels ont été retenus parce qu'ils sont investis d'une fonction référentielle spécifique pour ce qui est du choix de l'expression anaphorique et que la référenciation de leur expression anaphorique est capitale pour le procès.

- la sémantique seconde du verbe qui donne des informations sur la nature sémantique du référent à rechercher (animé vs inanimé, +/- humain...).

- la sémantique tertiaire qui correspond au sens lexical du verbe et à son rapport avec le contexte situationnel. » $(C f$. Dupuy-Parant, à paraître).

${ }^{17}$ Il pourrait être envisagé de tenir compte du statut thématique de «le bon homme » et «son brouet » (cf. Givón, 1983, Grosz, 1995 et Gundel, 1993). En effet, si l'on suppose que l'énoncé se continue ainsi : «le bon homme glissa et il tomba sur les genoux de la dame », selon la continuité thématique et les règles valentiello-référentielles, on serait face à une situation référentielle qui suggèrerait que le référent "bon homme » est celui repris par le pronom personnel il. Ainsi, pour éviter l'ambiguïté référentielle, si $i l$ devait reprendre le référent "brouet », une anaphore nominale serait plus sûre : «le bon homme glissa et le brouet tomba sur les genoux de la dame ». Cependant, pour le choix de l'expression anaphorique entre deux référents de nature sémantique différente, la sémantique verbale semble complémentaire et prépondérante sur le statut thématique. La concurrence peut naître dans un tel exemple parce que le verbe tomber est un verbe qui peut admettre comme agent sémantique un référent teinté du trait « $+/$ - personnel ». Si la construction avait été réalisée au moyen du verbe couler/se répandre, dont l'agent sémantique est teinté du trait «- personnel », cette ambiguïté n'existerait pas : «le bon homme glissa et il coula/se répandit sur les genoux de la dame » ( $c f$. Dupuy-Parant, à paraître).

${ }^{18}$ Extraits QJM : comparaisons : première joye, p. 11, 1. 188-189; tierce joye, p. 25, 1. 265 ; quinte joye, p. 36, 1.101 à $104 ;:$ quinte joye, p.44, 1. 402-403; quarte joye, p. 27, 1.1 à 18 ; métaphores : première joye, p. $6,1.1$ à 27 ; première joye, p. 13, 1. 252-253; seconde joye : p. 17, 1. 97-98; quinte joye, p. 36, 1. 109 à 113 ; quinte joye, p. 38, 1. 184-186; comparaison et métaphore : quarte joye, p. 28, 1. 32 à 48.

${ }^{19}$ Extraits de comparaison ne donnant pas suite à une chaîne anaphorique : Chr., livre I, chap. CCXLVII, p. 811, 1. 37 à 39: Or ... chapitainne, [...] ; Chr., livre I, chap. CCLVI, p. 851, 1. 37-41: Chil qui passerent parmi l'ost d'Engleterre, ... aillours. ; Mém., chap. VI, p. 242, 1. 26-30: De cela ... Henry. Dans cet extrait, les référents comparés sont tous deux patients des verbes, aymoit pour le référent ceste lignee de Lanclastre et aymoit sousentendu dans la proposition comparative, pour le référent celle d'Yort; Mém., chap. VIII, p. 255, 1. 23-27 : En ceste ... rompre. Dans cet extrait le sujet également agent sémantique du verbe travailloit est simplement éloigné de ce verbe par l'interposition d'une proposition relative - qui enchâsse elle-même une proposition conjonctive coordonnée à une seconde proposition relative qui enchâsse la proposition subordonnée comparative.

${ }^{20}$ Règles valentiello-référentielles : Un verbe ne peut avoir deux arguments de même identité référentielle (sauf verbes pronominaux réfléchis et constructions avec certaines prépositions; $c f .2003$ et 1998). La succession des verbes forme un chaînage valentiel reliant entre eux les rôles valentiels identiques (agents sémantiques entre eux; patients avec patients); excepté à la voix passive (relation valentiello-référentielle inversée). Ainsi, une identité référentielle maintenue dans l'un de ces deux rôles d'un verbe à l'autre sera reprise par anaphore pronominale ou zéro tandis qu'un changement d'identité référentielle dans la chaîne valentielle de l'agent sémantique ou du patient sera marqué par l'anaphore nominale.

Règle syntactico-sémantique (Dupuy-Parant, 2006, chap. 6 et p. 306) : Elle contraint ces dernières règles en fonction de la nature syntaxique de la proposition dans laquelle entre le verbe. Toutes les propositions reçoivent l'influence de la distribution du verbe qui les précède, mais seules les propositions non régies, temporelles et relatives en lequel laissent la distribution de leur verbe influer sur celle du verbe suivant. On parle alors de frontière de sortie poreuse par opposition aux autres propositions subordonnées (dont les comparatives) qui ont une frontière de sortie étanche puisqu'elles ne permettent pas au système valentiello-référentiel de leur verbe d'influer sur le système valentielloréférentiel du verbe suivant. N.B. : Les propositions régies enchâssées dans une proposition régie se conforment au fonctionnement syntactico-valentiel de la proposition enchâssante.

${ }^{21}$ Les référents au féminin singulier "L'armee dudict duc de Bourgongne" et "celle du Roy et dudict conte ensemble" entrent en comparaison et en inter-définition avec un référent, masculin singulier pour l'un " $d u c d e$ Bourgongne" et masculin pluriel pour l'autre "du Roy et dudict conte ensemble", par l'intermédiaire du complément de détermination. Le dénominateur commun à ces deux référents féminins étant l'armee et le référent $L$ 'armee dudict duc de Bourgongne étant agent sémantique du verbe estoit en proposition non régie, il semble que par simplification du facteur commun ' $L$ 'armee" pour ces deux référents féminins, le référent masculin singulier 'duc de Bourgogne' accède au rôle d'agent sémantique par anaphore pronominale avec le pronom personnel, $i l$, sujet du verbe avoit prins 
de la proposition non régie suivant la proposition comparative sans qu'il y ait de C.Rf. entre les deux référents comparés.

${ }^{22}$ Cette métaphore apparaît sous la forme nominale Ung mal ne ung peril qui réfère contextuellement à la fuite du roi Edouard d'Angleterre puis à sa prise en chasse par des bateaux ennemys des Angloys.

${ }^{23}$ Dans cet extrait, le référent "les affaires ou brouilliz de ce royaulme" est repris par les pronoms personnels de personne 6, ilz. Puis, le contexte précédent, qui renvoie à l'idée de guerre, est métaphorisé par ceste feste. Il s'agit donc d'une métaphore in abstentia filée, prolongée par le sémantisme du verbe conviéz (champ lexical de la fête).

${ }^{24}$ Références des quatre autres extraits qui fonctionnent comme (12) : QJM : Première joye, p. 13, 1. 252-256: Ainxin ... ses jours. ; Seconde joye, p. 17, 1. 97-99: Or est il en la nasse ... haste.; Quinte joye, p. 36, 1. 110 à 113 : [métaphore de l'amour avec l'amant comme l'on boit un bon vin vs l'amour avec le mari] : Auxi sachez ... temps. ; Quinte joye, p. 38, 1. 181-186 : [cf. extrait précédent] : Et le bon homme travaille ... le bon ypocras que elle a autreffoiz eu; Chr. (1 occ.) : livre I, chap. CCXLVII, p. 817-818, 1. 239-245: Se la contesse de Montfort, ... esquiers [le référent la fenme a messire Carle de Blois entre en relation avec le référent in absentia, 'le cheval'.].

${ }^{25}$ Références des synecdoques dans les Mém., livre III : la couronne : chap. I, p. 219, 1.24 ; chap. IV, p. 232, 1. 4 ; chap. VIII, p. 255, 1. 29, 1. 31, et p. 256, 1.4 ; son esperit : chap. I, p. 219, 1.4 ; sa personne : chap. 5, p. 240, 1. 7.

${ }^{26}$ Mém., livre 3, chap. 1, p. 219, 1. 6-10 où le référent métonymisant plusieurs villes apparaît en DI milieu discursif qui a ses spécificités propres.

${ }^{27}$ Occurrences pour lesquelles le référent métonymique apparaît dans le rôle de patient : Mém., livre III, Chapitre IV, p. 230, 1. 16 - p. 231, 1. 1: Comme ... batailles ; Mém., livre III, Chapitre IV, p. 231, 1. 33-36: Revenant au roy Edouard ... celle de Lenclastre. L'anaphore nominale de ces deux extraits est due à une possible C.Rf. entre les deux référents métonymiques co-présents : 'la maison de Lenclastre' et 'la maison d'Yort'. 